\title{
Wohin wird die Reise gehen? \\ Anforderungen einer zukunftsorientierten Schule an eine nachhaltige Medienbildung als Teil der Lehrerausbildung von heute ${ }^{1}$
}

\author{
Horst Niesyto
}

In den letzten Jahren hat die Mediennutzung bei Kindern und Jugendlichen insbesondere im Bereich digitaler Medien erheblich zugenommen und verdrängt bei einzelnen Gruppen bereits das bisherige Leitmedium Fernsehen. ${ }^{2}$ Kinder und Jugendliche eignen sich selbständig Medienangebote an, werden Mitglied in verschiedenen Medien-Communities, bilden eigene Vorlieben aus und demonstrieren so ihre Zugehörigkeit zu bestimmten Szenen. Die massenhafte Nutzung von E-Mails, Chatrooms, virtuellen Plattformen verändert Kommunikationsstrukturen und bietet neue Möglichkeit für Informationsbeschaffung, Lernen, Orientierung, Selbstausdruck und Kommunikation. Sie birgt aber auch Risiken, die von problematischen Medienangeboten über soziale Benachteiligung in der Mediennutzung bis hin zu fahrlässigen Formen des Umgangs mit Medien reichen. ${ }^{3}$ Gerade der Umgang mit persönlichen Daten in der Internetkommunikation offenbarte hier in letzter Zeit gravierende Fehlentwicklungen.

Menschliche Kommunikation ist untrennbar mit Medien verbunden. Alle zentralen Fragen unserer Zeit werden über Medien kommuniziert. Die Medienpädagogik geht davon aus, dass Medien als Teil der Lebenswelt der heranwachsenden Generationen positive Ressourcen zur Verfügung stellen, deren Nutzung in der heutigen Gesellschaft für alle Bürgerinnen und Bürger notwendig ist, die am Leben der Gesellschaft teilhaben wollen. Einfache Erklärungsmodelle zur Medienwirkung, eine pauschale Medienschelte oder der Versuch, Medien aus dem Alltag von Kindern und Jugendlichen zu verbannen, helfen nicht weiter. Sinnvoll und notwendig ist eine deutliche Verstärkung von medienpädagogischen Aktivitäten, um Kindern, Jugendlichen und Eltern bei einem verantwortungsvollen Umgang mit Medien zu helfen.

\footnotetext{
${ }^{1}$ Vortrag am 5. Juni 2008 in Frankfurt/Main auf der Fachveranstaltung „Lehren und Lernen in der digitalisierten Bildungswelt von morgen - eine Herausforderung an die Lehrerausbildung von heute“. Die Veranstaltung wendete sich an die Leiterinnen und Leiter der hessischen Studienseminare und wurde vom AfL-Projekt „Medien und Module“ im Rahmen der Landesinitiative „Schule@Zukunft“ (Kultusministerium Hessen) durchgeführt.

${ }^{2}$ Medienpädagogischer Forschungsverbund Südwest (Hg.) (2007): JIM-Studie 2007 Jugend, Information (Multi-) Media. Stuttgart: Landesanstalt für Kommunikation.

${ }^{3}$ Niesyto, Horst (2007): Mediensozialisation, gesellschaftliche Medienentwicklung und Medienkritik. In: Schnell, Ralf (Hg.): Medien: Nutzung - Medienwirkung - Medienregulierung. Themenheft der Zeitschrift für Literaturwissenschaft und Linguistik, Nr. 146, S. 23-46.
} 


\section{Wozu Medienbildung?}

\section{Problem der Optionenvielfalt und der sozial verantwortlichen Auswahl}

Die Digitalisierung führte zu einer Potenzierung von Informationen und Medienangeboten. Die klassische Funktion der Massenmedien, gesellschaftliche Komplexität zu reduzieren, damit verstehbar zu machen, hat heute angesichts vielfältiger konkurrierender Angebote an Bedeutung verloren. Das Internet gewinnt immer größere Bedeutung für Information, Kommunikation und die Beschaffung verschiedenster Angebote. Neben den damit verbundenen Chancen und Bildungspotenzialen sind aber die Risiken nicht zu unterschätzen. So sind im Internet trotz diverser Filter- und Schutzsysteme viele entwicklungsbeeinträchtigende und jugendgefährdende Angebote frei zugänglich. Ein restriktiver Kinder- und Jugendmedienschutz kann dieses Problem aber nur begrenzt lösen. Für eine freie und demokratische Gesellschaft gibt es vor allem einen Weg: auf Information, Bildung, Aufklärung der jungen Menschen zu setzen und sie durch Medienbildung zu befähigen, selbständig und sozial verantwortlich aus der Optionenvielfalt auszuwählen und Medien konstruktiv für die eigene Lebensgestaltung zu nutzen.

\section{Medien im Spannungsfeld von Markt und öffentlichem Kulturgut}

Medien sind einerseits ein wichtiger Wirtschaftsfaktor, sie sind aber auch ein öffentliches Kulturgut. Es kann nicht darum gehen, nur um der Quote und der Rendite willen alles auf den Markt zu bringen. Der Verlust an Programmqualität und eine schleichende Verletzung von Menschenwürde in diversen Formaten sind bedenkliche Entwicklungen, die nicht zu akzeptieren sind. Im Interesse einer humanen Mediengesellschaft ist es wichtig, hier Gegenmaßnahmen zu ergreifen. Ohne das im Grundgesetz verbriefte Recht zur freien Meinungsäußerung und das Recht auf künstlerische Ausdrucksfreiheit anzutasten, ist sicherzustellen, dass die bestehenden Regelungen im Kinder- und Jugendmedienschutz konsequent angewandt werden. Die Medienschaffenden sind verstärkt in die Verantwortung zu nehmen. Der öffentliche Diskurs über Programmqualität ist zu verstärken. Qualitätsbezogene Medienangebote brauchen Mediennutzer, die Programm- und Medienqualität erkennen können. Die Förderung von Medienkompetenz und Medienbildung ist deshalb für eine demokratische Gesellschaft unverzichtbar. 
Medienerziehung in Familie, Kindergärten, Schule und im außerschulischen Bereich

Es ist Aufgabe der Eltern, ihre Kinder auch im Medienumgang verantwortungsvoll zu erziehen und ihre Entwicklung in sozial verantwortlicher Weise zu fördern. Viele Eltern sind heute angesichts der rasanten Medienentwicklung verunsichert und überfordert. Auch gibt es nicht wenige Familien, in denen kaum Medienerziehung stattfindet und Eltern in ihrem eigenen Medienverhalten keine guten Vorbilder sind. Dies ist besonders in bildungsbenachteiligten Sozialmilieus ein Problem.

In Kindergärten spielt bis jetzt Medienerziehung so gut wie keine Rolle, obwohl viele Kinder bereits im vorschulischen Alter umfangreiche Medienerfahrungen haben. In der Schule sind zwar in den Bildungsplänen Ansatzpunkte für Medienerziehung und Medienbildung genannt. Es fehlt aber an der Konkretisierung für Fach- und Schulcurricula und es fehlt eine für alle Lehramtsstudierende verbindliche medienpädagogische Grundbildung.

Im außerschulischen Bereich gibt es zwar eine Reihe von Tätigkeiten, auch im Bereich der aktiven Medienarbeit. Die Mehrzahl der Aktivitäten ist aber von punktuellen Projektgeldern abhängig - es mangelt an strukturellen Finanzierungsmodellen, an kontinuierlichen Fort- und Weiterbildungsangeboten und an einer auf Langfristigkeit angelegten Kooperation mit schulischer Bildung.

Schule hat die Möglichkeit, einen verantwortlichen Medienumgang aufzuzeigen und alle Kinder zu erreichen. Sie hat insbesondere die Chance, Kinder und Jugendliche aus bildungsbenachteiligten Sozialmilieus zu fördern. Die entsprechenden Lernarrangements sollten sich nicht einseitig auf die Behebung von Defiziten konzentrieren, sondern auf die Stärkung von Selbstwirksamkeitserfahrungen abzielen. Schule ist gut beraten, ihren Bildungsauftrag in umfassendem Sinn als Lern- und Lebensort für Kinder und Jugendlichen zu begreifen. Es geht also nicht nur um Wissensaneignung für berufliche Perspektiven, es geht stets auch um persönlichkeitsbezogene Bildungsprozesse, die Kinder und Jugendliche darin unterstützen, altersspezifische Orientierungs- und Entwicklungsaufgaben zu bewältigen. Gerade im Jugendalter nehmen Orientierungs- und Identitätsbildungsprozesse eine große Bedeutung ein. Die kritische und reflexive Auseinandersetzung mit Medienvorbildern ist dabei eine wichtige Aufgabe. 


\section{Zum Stand der Qualifikation von Lehrkräften}

Erklärungen und Empfehlungen der Kultusministerkonferenz in den 1990er Jahren, die Aufnahme einzelner medienbezogener Inhaltsbereiche in Bildungspläne und diverse bundesweite und regionale Kampagnen zur besseren Verankerung von Medienbildung an Schulen brachten in den vergangenen 10 bis 15 Jahren durchaus Fortschritte. Dennoch besteht Handlungsbedarf. In der Breite gesehen ist Medienbildung an sehr vielen Schulen noch nicht angekommen. ${ }^{4}$ Es besteht eine Kluft zwischen allgemeinen bildungspolitischen Proklamationen („Medienkompetenz ist eine zentrale Schlüsselqualifikation“) und der konkreten Alltagspraxis. Mit Blick auf die Gesamtheit der Schulen sind Anstrengungen, medienpädagogische Inhalte in schulinternen Curricula fest zu verankern, eher Leuchtturmprojekten vorbehalten. Hier könnten von der aktuellen Diskussion zur Schulreform (Stichwort Ganztagsschulen) neue Impulse ausgehen. Ein besonderer Bedarf besteht an Grundschulen und in der Sekundarstufe I.

In der Lehrerausbildung (1. Phase) gibt es zwar an einzelnen Hochschulen und Bundesländern Anstrengungen, Medienpädagogik besser in den Prüfungs- und Studienordnungen zu verorten. Die verbindliche Verankerung einer medienpädagogischen Grundbildung in der Lehrerbildung ist aber nach wie vor noch eine entscheidende Zukunftsaufgabe. Es mangelt an der Realisierung fächerübergreifender Konzepte und auch an der Bereitschaft der Schulpädagogik, den Lehramtsstudierenden über einzelne mediendidaktische Ansätze hinaus eine grundlegende Medienbildung zu ermöglichen. Die Trennung von Mediendidaktik und Medienerziehung, eine einseitige Orientierung auf E-Learning-Initiativen und eine nach wie vor unterentwickelte Praxis- und Projektorientierung sind vielerorts hemmende Faktoren.

Medienbildung an Schulen ist in hohem Maße abhängig von den Medienkompetenzen der Lehrkräfte. Mehrere Studien wie etwa vom Medienpädagogischen Forschungsverbund Südwest zur Computernutzung und -kenntnisse von Lehrern/innen ${ }^{5}$ oder ein bilanzierender Bericht der Universität Paderborn zum Programm Schulen ans Netz ${ }^{6}$ verdeutlichen, dass es an praktischen Medienkompetenzen bei Lehrkräften häufig mangelt. Auch fehlen in diesem Bereich Evaluationsstudien und Programme zur Qualitätssicherung.

\footnotetext{
${ }^{4}$ Tulodziecki, Gerhard (2006): Schulische Medienpädagogik - von den 1950er Jahren bis heute. In: medien + erziehung, 50. Jahrgang, Nr. 5, Oktober 2006, S. 49-56.

${ }^{5}$ Medienpädagogischer Forschungsverbund Südwest (2003): Lehrer/-innen und Medien 2003. Nutzung, Einstellungen, Perspektiven. Baden-Baden.

${ }^{6}$ Herzig, Bardo/ Graf, Silke (2007): Digitale Medien in der Schule. Standortbestimmung und Handlungsempfehlungen für die Zukunft. Studie zur Nutzung digitaler Medien in allgemein bildenden Schulen in Deutschland. Universität Paderborn.
} 
Im Einzelnen sind folgende Bereiche zu nennen, in denen Lehramtsstudierende bislang keine verbindliche medienpädagogische Grundbildung erwerben:

- Kenntnisse über die Mediennutzung von Kindern und Jugendlichen und Qualifikationen für einen differenzierte Analyse und Bewertung von Chancen und Risiken der gesellschaftlichen Medienentwicklung;

- Kenntnisse und Qualifikationen für eine pädagogisch sinnvolle Medienkritik;

- Kenntnisse und Qualifikationen für einen didaktisch sinnvollen Einsatz von (digitalen) Medien und Formen aktiver Medienarbeit / Mediengestaltung (incl. dafür notwendiger medientechnischer und medienästhetischer Kenntnisse);

- Kenntnisse und Qualifikationen, um Medienbildung strukturell in Schulcurricula durch Kooperationen innerhalb und außerhalb der Schule zu verankern.

Wie lässt sich eine Qualifizierung in diesen Themenbereichen realisieren?

Zunächst geht es darum, dass Lehramtsstudierende sich (selbst)reflexiv mit der eigenen Mediennutzung und Medienbiographie auseinandersetzen, eigene mediale Präferenzen, Nutzungsbedürfnisse und Praktiken hinterfragen und die entsprechenden Präferenzen, Nutzungsbedürfnisse und Praktiken der Kinder und Jugendlichen kennen lernen. Gerade unter dem Aspekt sozialer und bildungsmäßiger Benachteiligung ist pädagogisch eine oberflächlichkritische Abwehrhaltung ${ }^{7}$ gegenüber Medien fatal, weil sie Kinder und Jugendliche nicht darin unterstützt, Medien für die eigene Persönlichkeitsbildung aktiv und differenziert zu nutzen. Gefragt ist eine pädagogische Begleitung, die Kindern und Jugendlichen Wege aufzeigt, mediale Aussagen bezüglich Aufmachung, Inhalt und damit verbundenen Werten und Normen zu hinterfragen. Eine Voraussetzung hierfür ist es, dass die angehenden Lehrkräfte die Medienpraxis der Kinder und Jugendlichen kennen - und eben nicht vorschnell Urteile abgeben. Es ist notwendig, an den Medienerfahrungen der Schülerinnen und Schüler anzuknüpfen und altersspezifische Formen der Medienkritik zu entwickeln.

Ein weiterer Punkt betrifft das Verhältnis von Grundlagenvermittlung und fächerintegrativer Medienbildung. Konzepte zur fächerintegrativen Medienbildung wurden seit den 1980er Jahren immer wieder entwickelt und erprobt. Es ist richtig, an fachbezogenen Themenstellungen anzusetzen und einen sinnvollen Medieneinsatz und Aufgaben der Medienerziehung und Medienbildung hierin zu verorten. Gerade ein Fach wie Deutsch bietet hier zahlreiche Anknüpfungspunkte und hat auf der theoretisch-konzeptionellen Grundlage eines erweiterten Text-

\footnotetext{
${ }^{7}$ Vgl. hierzu: Kommer, Sven (2006): Zum medialen Habitus von Lehramtsstudierenden. Oder: Warum der Medieneinsatz in der Schule eine so 'schwere Geburt' ist. In: Treibel, Annette/Maier, Maja S./Kommer, Sven/Welzel, Manuela (Hg.): Gender medienkompetent. Medienbildung in einer heterogenen Gesellschaft. Wiesbaden: VS-Verlag, 165-177.
} 
begriffs in den letzten Jahren einige Fortschritte erzielt. Dennoch bleibt festzuhalten, dass die große Mehrzahl von Lehramtsstudierenden heute die Hochschule verlässt, ohne eine medienpädagogische Grundbildung im Rahmen der studierten Fächer erhalten zu haben. Viel hängt von dem jeweiligen Fach- und Hochschulprofil ab, inwieweit medienerzieherische, mediendidaktische und mediengestalterische Aspekte Bestandteil der Ausbildung sind.

Nach meinen Beobachtungen und Erfahrungen bedarf es einer medienpädagogischen Grundbildung, die im Zusammenwirken von Grundlagenfächern (wie Erziehungswissenschaft) und den einzelnen Fachdidaktiken zu realisieren ist. Aufgabe der Grundlagenfächer ist es vor allem, Kenntnisse und Qualifikationen in den Bereichen Mediennutzung und Mediensozialisation von Kindern und Jugendlichen, gesellschaftliche Medienentwicklung (Chancen und Risiken), Entwicklung von medienkritischen und medienethischen Kriterien für die Beurteilung von Medienangeboten und eigenem Medienhandeln sowie Grundlagen zu Mediendidaktik und Mediengestaltung zu vermitteln. Die Fächer sollten mediendidaktische, mediengestalterische und medienerzieherische Bezüge auf dem Hintergrund fachspezifischer Themenzugänge herstellen und vor allem in projektbezogenen Arbeitsformen Medienwissen in unterschiedlichen Bereichen vertiefen. Damit dies möglich ist, benötigen die Studierenden ein medienbezogenes Grundwissen, das über fachspezifische Themenzugänge hinausreicht.

Das Dilemma der Medienbildung besteht institutionell darin, kein eigenes Schulfach zu sein, gleichzeitig aber Medienkompetenzen im Sinne von „Schlüsselqualifikationen“ zu vermitteln. Da die bisherige Erfahrung zeigt, dass Medienkompetenzen in den meisten Fächern rudimentär vermittelt werden, bedarf es neuer Konzepte, die im Zusammenwirken verschiedener Fächer eine medienpädagogische Grundqualifikation in der Lehramtsausbildung gewährleisten.

Ein dritter wichtiger Punkt ist das Verhältnis von 1. und 2. Phase der Lehrerbildung. In der 1. Phase der Lehrerbildung sollten wissenschaftliche Grundlagen zur Medienbildung, die bildungstheoretische Begründung und Verortung von Medienbildung, Einblicke in wichtige Medienstudien, die selbstreflexive Auseinandersetzung mit der eigenen Mediennutzung, das Kennenlernen mediendidaktischer und medienerzieherischer Konzepte, die exemplarische Entwicklung und Erprobung medienpädagogischer Unterrichtseinheiten in Schulpraktika, die Befähigung zur Entwicklung von Schulcurricula unter Berücksichtigung von Medienbildung und der Erwerb von Qualifikationen zur Selbstevaluation medienpädagogischer Praxis. Begleitend sind medientechnische und medienästhetische Seminare und Kurse anzubieten, die insbesondere Grundlagen im Arbeiten mit digitalen Medien vermitteln. In der 2. Phase der Lehrerbildung sollte es vor allem darum gehen, das erworbene medienpädagogische Grundla- 
genwissen in fächerübergreifenden Seminaren zu vertiefen, in der Unterrichtsplanung den fachbezogenen Medieneinsatz und Aspekte der Medienbildung zum regelmäßigen Bestandteil von didaktisch-pädagogischen Überlegungen zu machen und Sicherheit im praktischen Umgang mit Medien zu gewinnen. Hierfür könnten die aktuellen Diskussionen zu Bildungsstandards in der Medienbildung hilfreich sein, wenn sie alters- und entwicklungsbezogen Minimalstandards für SchülerInnen definieren. Erste Überlegungen hierzu liegen vor, die hinsichtlich theoretisch-konzeptioneller Begründung, Komplexitätsgrad und Praktikabilität weiter zu diskutieren sind. ${ }^{8}$ Dabei wird nicht nur die Frage sein, ob Standards zur Medienbildung mehr nach Medienarten, Aufgabenbereichen bzw. Medienkompetenz-Dimensionen zu strukturieren sind. Übergeordnet ist die Frage, welche grundlegenden Ziele Medienbildung verfolgt und in welchem Verständnis von Bildung und Lernen sich Medienbildung verortet. Sach-, Methoden-, Sozial- und Medienkompetenzen können nicht unabhängig von schulischen Leitbildern entwickelt werden.

Eine Schule, die Lern- und Lebensort für Schülerinnen und Schüler sein möchte, muss sich für die Lebens- und Medienwelten der Kinder und Jugendlichen öffnen, ihre Bedürfnisse und Themen ernst nehmen und konsequent an vorhandenen Kompetenzen und Stärken anknüpfen. Nicht nur für die Primarstufe und die Sekundarstufe I gilt: Stärkere Integration anschaulicher, präsentativ-symbolischer Ausdrucks- und Kommunikationsformen, Förderung von Schriftspracherwerb und media literacy, Förderung von kognitivem und sozial-emotionalem Lernen. Formen der aktiven Medienarbeit/Mediengestaltung bieten hierfür sehr gute Möglichkeiten. Die eigene Artikulation mit unterschiedlichen Medien wird in Zeiten von Internet und Web 2.0 immer wichtiger. LehrerInnen sollten SchülerInnen motivieren, Medien aktiv und kreativ für Bildungs- und Lernprozesse, für Selbstausdruck und Kommunikation zu nutzen. Gerade für Kinder und Jugendliche aus sog. bildungsfernen Milieus liegen hierin Chancen für mehr Partizipation.

\section{Medien, Bildungsbenachteiligung und Chancen aktiver Medienarbeit}

Die Auseinandersetzung mit dem Themenfeld „Medien und soziale Benachteiligung“ verweist aus medienpädagogischer Perspektive auf vielschichtige Problemstellungen. Als Kernpunkte lassen sich folgende Überlegungen festhalten:

\footnotetext{
${ }^{8}$ Vgl. die Beiträge von Gerhard Tulodziecki und Heinz Moser über „Standards in der Medienbildung“ in der Schriftenreihe des Paderborner Lehrerausbildungszentrums, Heft 16/2007.
} 
- Bezüglich des Zugangs zu digitalen Medien zeichnet sich in den letzten Jahren eine enorme Veralltäglichung insbesondere bei Jugendlichen ab. Dennoch ist nicht zu übersehen, dass im Sozialisationsfeld Schule nach wie vor schulartenspezifische Unterschiede bestehen z.B. eine schlechtere Ausstattung und Zugänge an Grundschulen und in der Sekundarstufe I. ${ }^{9}$

- Die Formen und Funktionen der Medienaneignung sind unterschiedlich und lassen Bezüge zu sozialen und bildungsbezogenen Kontexten erkennen. Dies betrifft vor allem Unterschiede in der Nutzung von mehr wissens- bzw. mehr unterhaltungsorientierten Angeboten $^{10}$ sowie Unterschiede bezüglich vorhandener Lese- und Schreibkompetenzen ${ }^{11}$ (auch im Hinblick auf Nutzungsmotivationen und Navigationspraxen im Internet ${ }^{12}$ ).

- Im Bereich der schulischen Medienbildung reicht es nicht aus, Lehrkräfte technisch und methodisch zu qualifizieren. Angesichts einer Kluft in den medialen Habitusformen zwischen vielen PädagogInnen einerseits und vielen SchülerInnen andererseits gilt es, „nicht nur die Medienkompetenz der Schülerinnen und Schüler und Auszubildenden, sondern auch die medienpädagogische Kompetenz der Lehrkräfte zu stärken“, um Blockaden in pädagogischen Prozessen zu verhindern. ${ }^{13}$

- Entgegen medienfixierten Konzepten bewahrheitet sich der alte Grundsatz, von den Lebenswelten der Kinder und Jugendlichen, ihren sozialen und kommunikativen Kontexten, ihren handlungsleitenden Themen auszugehen. Subjekt- und Lebensweltorientierung bedeutet auch, die vorhandenen Formen der Medienaneignung zu kennen und zu respektieren und die in professionell-pädagogischen Kontexten entwickelten Kompetenzkriterien nicht zu verabsolutieren.

- Die zuletzt genannten Punkte legen die Schlussfolgerung nahe, stärker an zielgruppenspezifischen Konzepten der Medienbildung zu arbeiten, um die vorhandenen Potenziale und Ressourcen von Kindern und Jugendlichen aus benachteiligten Milieus besser fördern zu können.

\footnotetext{
${ }^{9}$ Medienpädagogischer Forschungsverbund Südwest (Hg.) (2005): JIM-Studie 2005 Jugend, Information (Multi-) Media. Stuttgart: Landesanstalt für Kommunikation.

${ }^{10}$ Wagner, Ulrike / Theunert, Helga (Hg.) (2006): Neue Wege durch die konvergente Medienwelt. BLM-Schriftenreihe, Bd. 85. München: Fischer-Verlag.

${ }^{11}$ Groeben, Norbert/ Hurrelmann, Bettina (Hg.) (2002): Lesekompetenz. Bedingungen, Dimensionen, Funktionen. Weinheim und München: Juventa.

12 Iske, Stefan/ Klein, Alexandra/ Kutscher, Nadia (2004): Digitale Ungleichheit und formaler Bildungshintergrund - Ergebnisse einer empirischen Untersuchung über Nutzungsdifferenzen von Jugendlichen im Internet. URL: www.kibbielefeld.de/externelinks2005/digitaleungleichheit.pdf (Zugriff: 27.07.2008)

13 Treibel, Annette/Maier, Maja S. (2006): Gender medienkompetent? Eine Einleitung. In: Treibel, Annette/Maier, Maja S./Kommer, Sven/Welzel, Manuela (Hg.): Gender medienkompetent. Medienbildung in einer heterogenen Gesellschaft. Wiesbaden: VS-Verlag, 11-23.
} 
- Im Bereich der medienpädagogischen Forschung mangelt es nicht zuletzt an Studien in Hauptschul- und Migrationsmilieus. ${ }^{14}$ Dies betrifft sowohl Fragen der (prozessbezogenen) Medienaneignung und Mediensozialisation als auch die Untersuchung des vorhandenen Medieneinsatzes und der medienbildnerischer Aktivitäten in den entsprechenden pädagogischen Einrichtungen. An Hochschulen gibt es nur wenige empirische Studien zur Entwicklung von Medienkompetenzen und medienpädagogischen Kompetenzen im Studienverlauf (Lehramtsstudium; andere pädagogische Studiengänge).

In der Zusammenschau dieser Punkte wird deutlich, dass die Frage „Medien und soziale Benachteiligung“ primär kein Medienproblem ist. Medien transportieren einerseits Chancen und Potenziale für mehr selbstgesteuerte Aneignungs- und Bildungsprozesse. In Verbindung mit unterschiedlichen Ressourcen - zu denen wesentlich familiäre und schulische Erfahrungsfelder gehören - können sie aber auch vorhandene Unterschiede im Sinne von Bildungsbenachteiligung verfestigen. Medienbezogene Grundhaltungen und pädagogische Konzepte von Lehrkräften, die sich zu wenig den Lebens- und Medienwelten der Kinder und Jugendlichen öffnen und keine zielgruppenspezifischen Lernarrangements bieten, sind in diesem Zusammenhang ein benachteiligender Faktor.

Medienbildung ist gefordert, Gegengewichte zu Prozessen gesellschaftlicher Ausgrenzung zu setzen - z. B. um Kindern und Jugendlichen aus Hauptschul- und Migrationsmilieus Erfahrungen der Selbstwirksamkeit durch handlungsorientierte Formen der Mediengestaltung zu ermöglichen. Vor allem in der praktischen Auseinandersetzung mit Medien kann es gelingen, Fähigkeiten zur reflexiven Distanz gegenüber medialen Inszenierungen in der Kombination unterschiedlicher Komponenten auszubilden, wobei an Formen alltäglicher Medienkritik angeknüpft werden kann. Gleichzeitig sollte Medienbildung darauf achten, nicht in eine Modernisierungsfalle zu tappen, die einseitig kognitive, auf Wissenserwerb orientierte Konzepte favorisiert und die sozial-kommunikativen und sozial-ästhetischen Dimensionen von Kompetenzbildung unterschätzt. Dies bedeutet, dass Medienpädagogen genau hinschauen sollten, wie Kinder und Jugendliche aus benachteiligenden Verhältnissen mit traditionellen und neuen Medien umgehen, welche Form des Zugangs, der Aneignung und des Ausdrucks sie bevorzugen und entwickeln.

\footnotetext{
${ }^{14}$ Erfreulicherweise startete 2006 ein Forschungsprojekt zum Thema „Bildungsbenachteiligung und multifunktionale Medien“, das vom JFF - Institut für Medienpädagogik (München) mit Förderung des Bundesministeriums für Bildung und Forschung (BMBF) bis Ende August 2008 durchgeführt wird. Hauptzielgruppe sind Hauptschüler/innen.
} 
Auf dem Hintergrund vieljähriger Berufspraxis und verschiedener Studien möchte ich folgende Punkte hervorheben:

- Erfahrungs- und Lebensweltorientierung: Anknüpfen an den vorhandenen Stärken und an den vorhandenen Themen, die Kinder und Jugendliche haben und die für sie zentral und handlungsleitend sind.

- Bilder, Musik, Körpersprache stärker integrieren. Dies ist besonders für Kinder und Jugendliche wichtig, die Schwierigkeiten mit der Wort- und Schriftsprache haben. Aus den Studien über Bildungsbenachteiligung die Schlussfolgerung zu ziehen, Kindern und Jugendlichen aus benachteiligenden Verhältnissen verstärkt schriftsprachliche Kompetenzen zu vermitteln, reicht nicht aus. Notwendig ist ein integriertes Konzept, das wort- und schriftsprachliche mit bildhaften und multimedialen Ausdrucks- und Kommunikationsformen in eine Balance bringt.

- Ästhetische Reflexivität fördern: Mit der Integration medialer Kompetenzen verbinden sich auch Formen einer anderen Reflexivität. Dies bedeutet z.B. im Bereich audiovisueller und multimedialer Produktion, von den selbst erstellten Aufnahmen auszugehen, diese gemeinsam anzusehen, zu vergleichen und sich gegenseitig Verbesserungshinweise zu geben. Ausgangspunkt und Gegenstand von Reflexionen sind die (audio)visuellen Materialien und die damit verbunden Ausdrucksintentionen; sinnliche Wahrnehmung und reflexive Verarbeitung sind als Symbolverstehen miteinander verknüpft.

- Medienästhetisch-kulturelle Kompetenzen sind auch für die Arbeitswelt wichtig: Jugendliche haben erst einmal das Bedürfnis, für eigene Medienproduktionen Themen aufzugreifen, die sie insgesamt beschäftigen. Die dabei erworbenen Kompetenzen sind aber auch für die Arbeitswelt wichtig: sozial-kommunikative, ästhetische, technische, methodische Kompetenzen. Diese Verknüpfungsmöglichkeiten von „ästhetisch-kulturell“ und „arbeitsweltbezogen“ sollte man konzeptionell viel stärker im Auge haben - anstatt einseitig auf technisch-instrumentelle Medienkurse zu setzen.

- Spielerische und non-lineare Arbeitsweisen fördern: Heute leben wir in einer Zeit, in der wir mit einer enormen Fülle verschiedenartiger Informationen konfrontiert sind. Daraus ergibt sich die Forderung, eigene Kriterien für die Auswahl zu entwickeln und gleichzeitig für unterschiedliche Perspektiven offen zu sein, um eigene Horizonte zu erweitern. In pädagogischen Kontexten hat sich hierfür ein Mix aus spielerischen Arbeitsformen, einem Rahmen und situationsspezifischen Inputs bewährt. „Rahmen“ meint, eine gewisse Struktur bieten, aber auch genügend Zeit und Flexibilität, die eine Beweglichkeit im Sinne von 
Experimentieren ermöglicht. Hierzu gehören auch kleinschrittige Übungsaufgaben und Produktionsmöglichkeiten, die weder unter- noch überfordern.

- Präsentation und Kommunikation lernen: Dies sind sog. Schlüsselkompetenzen, die immer wichtiger werden: nicht nur zu produzieren, sondern das selbst Gestaltete anderen zu zeigen und vorzustellen, Feedbacks zu erhalten, zuzuhören, auf andere einzugehen. Das Präsentieren befähigt, zu dem eigenen Produkt zu stehen, Kritik auszuhalten, auf Kritik einzugehen. Dies ist ein Erfahrungswert aus vielen Projekten: durch das Präsentieren selbst erstellter Medienproduktionen Selbstbewusstsein gewinnen und ermutigt werden, die eigene Arbeit fortzusetzen.

\section{Zusammenfassung: Anforderungen an eine nachhaltige Medienbildung}

Die Herausforderung besteht darin, von einer Phase der Modellprojekte und verschiedenen Einzelprojekten auf lokaler und regionaler Ebene zu einer Phase struktureller Veränderungen überzugehen. Einzelne Modellprojekte und Förderungen sind wichtig, reichen aber nicht aus. Es ist einfach ein krasser Missstand, dass es in der Breite gesehen keine Medienbildung an Schulen und Hochschulen gibt. Man hat über viele Jahre hinweg den Einfluss der Medien einfach unterschätzt - und zwar in alle Richtungen, kognitiv, emotional, die Sprache, die Bilder, die Phantasie betreffend. Anstatt ,Medienverwahrlosung’ zu beklagen und Gesetze zur Verschärfung des Jugendmedienschutzes zu fordern gilt es, die Medienbildung in der Gesellschaft zu verstärken! Medienpädagogik muss dauerhaft in verschiedenen Bildungsbereichen verankert werden.

Für eine Medienbildung mit Kindern und Jugendlichen benötigen wir Pädagoginnen und Pädagogen, die über eine Bandbreite von Medienkompetenzen, von Wissen über den Sozialisationshintergrund sowie über geeignete gruppenpädagogische und methodisch-didaktische Qualifikationen verfügen. Wir brauchen in allen pädagogischen Berufen eine medienpädagogische Grundbildung - von der frühen Bildung über die Lehrerbildung bis zur Erwachsenenund Seniorenbildung, um ein Grundverständnis für Fragen der Mediensozialisation und der Möglichkeiten von Medienbildung zu schaffen. Medienbildung muss zu einem verbindlichen Bestandteil der Lehrerbildung in allen Phasen werden. Dabei gilt es, mehr Kohärenz zwischen der Medienbildung in den Fächern (Bildungsstandards; curriculare Umsetzung) und zwischen den verschiedenen Phasen der Lehrerbildung (wissenschaftliche Grundlagen und praxisrelevante Fragestellungen und Konzepte) zu schaffen. Hierzu gehört auch ein Wissen, wie im Kontext von lokalen und regionalen Netzwerken Personen und Ressourcen gezielt für be- 
stimmte Vorhaben zu aktivieren sind, die man aus eigenen Kräften nicht realisieren kann. Es gibt inzwischen eine Reihe regionaler und bundesweiter Online-Plattformen, verschiedene Modellprojekte und eine Vielzahl an medienpädagogischer Literatur. Woran es vor allem fehlt, ist die verbindliche Verankerung medienpädagogischer Inhalte in der Ausbildung von pädagogischen Fachkräften sowie schulnahe Fort- und Weiterbildungsmöglichkeiten.

Nach wie vor sind viele Lehrkräfte noch zu sehr in einer Symbolsozialisation befangen, die auf dem Diskursiven, auf dem Wort- und Schriftsprachlichen beruht. Oft haben sie Angst, sich auf bestimmte Gesten, Ausdrücke, körperliche Ausdrucksformen einzulassen, gehen zu thematisch und pädagogisch-funktional vor, anstatt erst einmal soziale Beziehungen herzu stellen. Das ist ein Punkt, der gerade für Kinder und Jugendliche aus benachteiligenden Verhältnissen sehr wichtig ist. Ihnen das Gefühl zu geben: sie werden akzeptiert, mit all dem, wie sie sind, einen Raum zu haben, dies auszuleben - und ihnen zugleich Möglichkeiten zu geben, neue Kompetenzen zu erwerben, neue Erfahrungen zu machen auf einer Grundlage, die gegenseitige persönliche Wertschätzung mit sozial-emotionalen, ästhetischen und inhaltlichen Lernprozessen verbindet.

\section{Hinweis zum Autor}

Horst NIESYTO, Dr. rer. soc., Diplompädagoge, Professor für Erziehungswissenschaft mit dem Schwerpunkt Medienpädagogik an der Pädagogischen Hochschule Ludwigsburg. Schwerpunkte in Forschung und Lehre: Ästhetisch-symbolische Fragen in Bildungsprozessen, Grundfragen und Konzepte der Medienpädagogik, interkulturelle Medienbildung, Medienpädagogik und soziokulturelle Unterschiede, Filmbildung, medienpädagogische Praxisforschung, visuelle Forschungsmethoden. Funktionen: Leiter der Abteilung Medienpädagogik, Sprecher der Kommission Medienpädagogik in der Deutschen Gesellschaft für Erziehungswissenschaft (DGfE), stellv. Mitglied in der Kommission Jugendmedienschutz (KJM), Mitglied in verschiedenen Fachbeiräten.

\section{Kontakt}

Prof. Dr. Horst Niesyto, Pädagogische Hochschule Ludwigsburg, Institut für Erziehungswissenschaft Abteilung Medienpädagogik, Reuteallee 46, D-71634 Ludwigsburg, Tel: +49 (0) 7141-140-221 E-Mail: niesyto@ph-ludwigsburg.de, Homepage: www.ph-ludwigsburg.de/5380.html 